AIAA-2002-0836

\title{
NUMERICAL SIMULATION OF SUPERSONIC FLOW AROUND WING-BODY CONFIGURATION WITH INTEGRATED ENGINE NACELLE
}

\author{
Masahiro Kanazaki ${ }^{*}$, Shigeru Obayashi ${ }^{\dagger}$ and Kazuhiro Nakahashi ${ }^{\ddagger}$ \\ Tohoku University, Sendai 980-8577, JAPAN
}

\begin{abstract}
This paper presents numerical simulation of flowfields around a supersonic transport aircraft with an integrated engine nacelle. In this study, flowfields were simulated by solving the Euler equations with the unstructured grid method for handling the complex geometry. To simulate intake flows at actual flights, a bump was introduced inside the nacelle. The effect of nacelle mass flow ratios on overall aerodynamic performance was investigated in detail by changing bump heights. The spillage drag was calculated and found to have a large impact on the total drag. Computed results were compared with wind tunnel data obtained at National Aerospace Laboratory of Japan.
\end{abstract}

\section{Introduction}

The scaled supersonic experimental aircraft with propulsion system is now under the design process by National Aerospace Laboratory of Japan (NAL) [1]. The preliminary configuration of the jet-powered experimental aircraft used for the wind tunnel test is shown in Fig.1. The design has to account for strong aerodynamic interactions among wing, fuselage, and nacelles.

The wind tunnel model has flow-through nacelles. To simulate actual flights, nacelle mass flow ratios have to be controlled because the spillage drag is expected to have an influence on the airplane's total drag. Because it will be very difficult to control the nacelle mass flow by simulating the actual turbo fan engine, a bump was introduced inside of the nacelle. The nacelle mass flow ratios will be controlled by changing the bump heights.

In this paper, the three-dimensional Euler equations were solved by using the unstructured grid approach. The bump is introduced by deforming the boundary grid inside the nacelle, and the field grid is deformed accordingly.

Aerodynamics performance of the wind tunnel

\footnotetext{
* Graduate Student, Institute of Fluid Science

${ }^{\dagger}$ Associate Professor, Institute of Fluid Science, Associate Fellow AIAA

${ }^{\ddagger}$ Professor, Department of Aeronautics and Space

Engineering, Associate Fellow AIAA
}

Copyright ( $\odot 2002$ by the American Institute of Aeronautics and Astronautics, Inc. All rights reserved. model will be calculated at various nacelle mass flow ratios and compared with experiment [2].

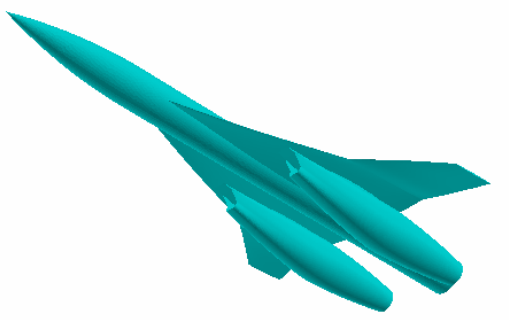

Fig. 1 Wind tunnel model 01 for scaled supersonic experimental aircraft designed at NAL

\section{Flow Solver}

In this study the flowfield was calculated by the Euler equations written as,

$$
\frac{\partial}{\partial t} \int_{\Omega} \mathbf{Q} d V+\int_{\partial \Omega} \mathbf{F}(\mathbf{Q}) \cdot \mathbf{n} d S=0
$$

where $\mathbf{Q}=[\rho, \rho u, \rho v, \rho w, e]^{T}$ is the vector of conservative variables; $\rho$ is the density; $u, v, w$ are the Cartesian velocity components; and $e$ is the total energy. The vectors $F(Q)$ represent the inviscid flux and $\mathbf{n}$ is the outward normal of $\partial \Omega$ which is the boundary of the control volume $\Omega$.

Equations (1) are solved by a finite-volume cell-vertex scheme and can be written in an algebraic form as follows

$$
\frac{\partial \mathbf{Q}_{i}}{\partial \mathbf{t}}=-\frac{1}{V_{i}}\left[\sum_{j(i)} \Delta S_{i j} \mathbf{F}(\mathbf{Q})_{i j} \cdot \mathbf{n}_{i j}\right]
$$

where, $\Delta S_{i j}$ is a segment area of the control volume boundary associated with the edge connecting points $i$ and $j$. The term $\mathbf{F}$ is an inviscid numerical flux vector normal to the control volume boundary, and $Q_{i j}^{ \pm}$are values on both sides of the control volume boundary. The subscript of summation, $j(i)$ refers to all node points connected to node $i$.

The Harten - Lax - van Leer - Einfeldt - Wada (HLLEW) Riemann solver [3] is used for the numerical flux computations. Second-order spatial accuracy is realized by a liner reconstruction of the primitive variables $\mathbf{q}=[\rho, u, v, w, p]^{T}$ inside the control volume as

$$
\mathbf{q}(x, y, z)=\mathbf{q}_{i}+\Psi_{i} \nabla \mathbf{q}_{i} \cdot\left(\mathbf{r}-\mathbf{r}_{i}\right)
$$

where, $\mathbf{r}$ is a position vector and $i$ is the node 
number. The gradient associated with the control volume centroid is volume-averaged gradient computed from the values in the surrounding grid cells. A limiter, $\Psi$, is used to make the scheme monotone. Here Venkatakrishnan's limiter [4] is used because of its superior convergence properties.

The lower-upper symmetric Gauss-Seidel (LU-SGS) implicit method [5], originally developed for the structured grid, is applied to compute the high Reynolds number flows efficiently. The LU-SGS method on the unstructured grid can be derived by splitting node points $j(i)$ into two groups, $j \in L(i)$, and $j \in U(i)$ for the first summation in LHS of Eqs. (2). With $\Delta \mathbf{Q}=\Delta \mathbf{Q}^{n+1}-\Delta \mathbf{Q}^{n}$, the final form of the LU-SGS method for the unstructured grid becomes the following two sweeps:

Forward sweep:

$$
\Delta \mathbf{Q}_{i}^{*}=\mathbf{D}^{-1}\left[\mathbf{R}_{i}-\sum \Delta S_{i j} \mathbf{A}_{j}^{-} \Delta \mathbf{Q}_{j}^{*}\right]
$$

Backward sweep:

$$
\Delta \mathbf{Q}_{i}=\Delta \mathbf{Q}_{i}^{*}-\mathbf{D}^{-1} \sum_{j(i)} \Delta S_{i j} \mathbf{A}_{j}^{-} \Delta \mathbf{Q}_{j}
$$

where,

$$
\mathbf{D}=\frac{V_{i}}{\Delta t} \mathbf{I}+\sum_{j(i)} \Delta S_{i j} \mathbf{A}_{i}^{+}
$$

The term $\mathbf{D}$ is diagonalized by the Jameson-Turkel approximation [6] of the Jacobian as $\mathbf{A}^{ \pm}=0.5\left(\mathbf{A} \pm \rho_{\mathbf{A}} \mathbf{I}\right)$, where $\rho_{A}$ is a spectral radius of Jacobian A .

The lower-upper splitting of Eqs. (4) for the unstructured grid is done by a grid reordering technique [5] that was developed to improve the convergence and the vectorization.

\section{Nacelle Mass Flow Control by Bump Heights}

\subsection{Bump Definition}

The bump is introduced in the engine nacelle to examine the influence of nacelle mass flow ratios on the total aircraft drag. The bump geometry is defined by an exponential function as,

$$
h_{\text {bump }}=\alpha \times \exp \left(-\beta \times x^{2}\right)
$$

where, $\alpha$ is a height of the bump and $\beta$ is an indicator of the width of the bump. The height and width are normalized by the length of the fuselage. In this study, $\beta$ is fixed at 0.095 . The bump is placed in the nacelle as shown in Fig. 3.

\subsection{Nacelle Mass Flow Control}

The nacelle mass flow ratios can be controlled by changing the bump heights. The mass flow into the nacelle $\dot{m}$ is written as,

$$
\dot{m}=\rho_{e} U_{e} A_{e}
$$

where, $\rho_{e}, U_{e}$ and $A_{e}$ are density, velocity and area at the exit of the nacelle, respectively. The mass flow $\dot{m}$ is normalized by the maximum mass flow into the nacelle $\dot{m}_{\max }$. The maximum mass flow $\dot{m}_{\text {max }}$ is written as,

$$
\dot{m} \max =\rho_{\infty} U_{\infty} A_{i}
$$

where, $\rho_{\infty}$ and $U_{\infty}$ are freestream density and velocity, respectively, and $A_{i}$ is the intake area projected to the front view of the nacelle. Then the nacelle mass flow ratio can be written as,

$$
\frac{\dot{m}}{\dot{m}_{\max }}=\frac{\rho_{e} U_{e} A_{e}}{\rho_{\infty} U_{\infty} A_{i}}
$$

The mass flow ratio can be controlled by changing the height of the bump as shown in Fig. 4. In this study, the bump heights were set to $0 \%, 24 \%, 32 \%$, $34 \%$ and $36 \%$ of the nacelle radius.

\subsection{Prediction of Spillage Drag}

When the nacelle mass flow is controlled, the spillage drag should be calculated. The spillage drag can be predicted by the conservation of the momentum [7] as follows,

$$
\begin{array}{r}
\int_{a b}\left(\rho u_{n} u_{x}+p_{\infty}\right) d s+\int_{b c} p_{x} d s= \\
\int_{c d}\left(\rho u_{n} u_{x}+p_{x}\right) d s
\end{array}
$$

where, $\rho, U_{x}$ and $p_{x}$ are density, velocity and pressure along the $x$ coordinate, respectively, $U_{n}$ is velocity normal to the boundary and $p_{\infty}$ is freestream pressure. Hence the equilibrium of forces is written as,

$$
\int_{a b} p_{\infty} d s+\int_{b c} p_{\infty} d s=\int_{c d} p_{\infty} d s
$$

Eqs. (10) and (11) give the equation written as,

$$
\begin{array}{r}
\int_{a b} \rho u_{n} u_{x} d s+\int_{b c}\left(p_{x}-p_{\infty}\right) d s= \\
\int_{c d}\left[\rho u_{n} u_{x}+\left(p_{x}-p_{\infty}\right)\right) d s
\end{array}
$$

The second term $\int_{b c}\left(p_{x}-p_{\infty}\right) d s$ corresponds to the spillage drag and $\rho u_{n}$ corresponds to the mass flow through a unit area normal to the boundary. It is still difficult to calculate the first integration $\int_{a b} \rho u_{n} u_{x} d s \quad$ because the point $b$ has to be determined from the streamline that will reach the point $c$ at every mass flow ratio as illustrated in Fig. 5. Considering the conservation of the mass flow one can obtain the relation written as,

$$
\int_{a b} \rho u_{n} u_{x} d s=u_{\infty} \int_{a b} \rho u_{n} d s=u_{\infty} \int_{c d} \rho u_{n} d s
$$

Using this equation, the spillage drag is rewritten as,

$$
D_{s p i l}=\int_{c d}\left[\rho u_{x}\left(u_{x}-u_{\infty}\right)+\left(p-p_{\infty}\right)\right] d s
$$




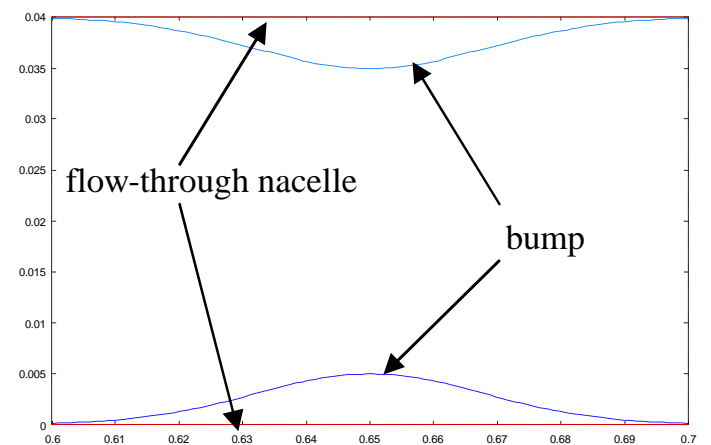

Fig. 2 Definition of bump by exponential function

(a)

(b)

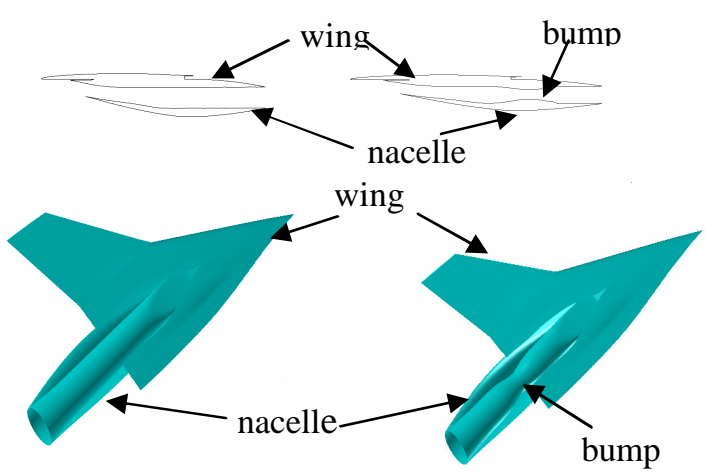

Fig. 3 Geometry of bump inside nacelle and cross sectional view of nacelle; (a)without bump, (b)with bump

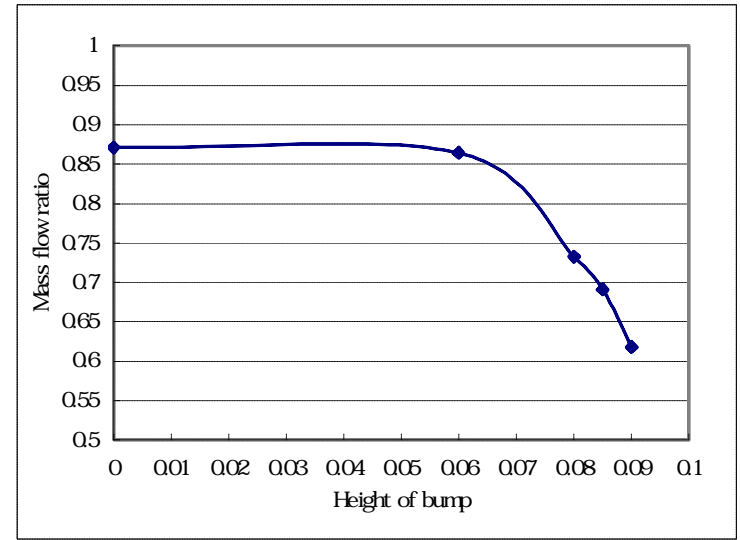

Fig. 4 Variation of mass flow ratios according to bump heights

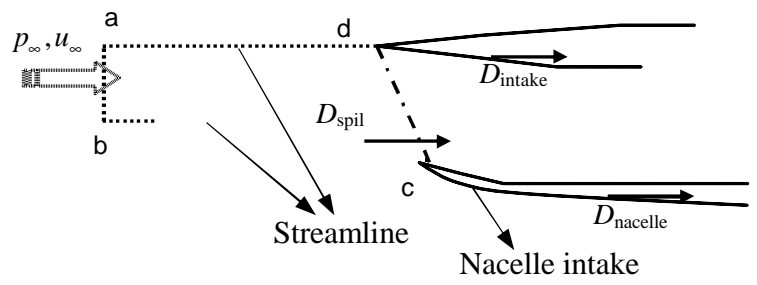

Fig. 5 Nacelle intake flow and streamlines

\section{Results}

The geometry used in the present study is the preliminary wind tunnel model for the scaled experimental supersonic airplane designed at NAL. Computational grid is shown in Fig. 6. The total number of the nodes and elements are about 910,000 and 5,100,000, respectively. Solutions were obtained at a freestream Mach number, $M_{\infty}=1.4$, and angle of attack, 0 deg.

\subsection{Computed Pressure Distributions}

Computed pressure contours on the wing-bodynacelle configuration with the $36 \%$ bump height case are compared with the flow-through nacelle case in Fig. 7. Strong shock waves can be found near the nacelle intake with the $36 \%$ bump case. The shock waves extend to the upper surface of the wing.

Effects of the bump on the pressure distributions are also illustrated in Figs. 8 and 9. Figure 8 shows the cross sectional view of the nacelle. Figure 9 shows the pressure distributions on the lower surface of the model. While the air is not compressed in the flow-through nacelle, the air is highly compressed in the nacelle with the bump as expected. In the latter case, the resulting subsonic region extends upstream of the inlet and it creates the strong shock wave.

\subsection{Variations of Aerodynamic Performances due to Nacelle Mass Flow Ratios}

Force measurements in the experiment were processed to exclude aerodynamic forces inside the nacelle because the preliminary estimate of the airplane performance is carried out as a sum of external aerodynamic force and propulsion-related force estimated separately. Following the experiment, the computed lift and drag also excludes forces inside the nacelle.

Lift and drag coefficients at various nacelle mass flow ratios are plotted in Figs. 10 and 11, respectively. In Fig. 10, the lift coefficient of the wing-body increases as the mass flow ratio decreases. This increase was caused by the shock wave near the intake of the nacelle as shown in Fig. 9. On the other hand, the lift coefficient of the nacelle decreases. The computations at $A_{o} / A_{i}=0.6$ and 0.9 converge well and steady shock waves are observed upstream of the inlet and inside the nacelle, respectively. The computations at $A_{o} / A_{i}=0.7$ and 0.8 do not converge very well.

Figure 11 shows the drag variation. As the nacelle mass flow ratio decreases, the drag coefficient of the nacelle decreases due to the reduction of the pressure behind the shock wave near the inlet. Because other drag components remain nearly constant, the wingbody-nacelle drag coefficient decreases slightly as the drag coefficient of the nacelle decreases.

Does the total drag decrease as the bump reduces the nacelle mass flow ratio, although the strong shock wave appears? It sounds inconsistent. This leads to the calculation of the spillage drag mentioned in Section 3.2. Figure 12 shows the spillage drag and the total drag coefficients compared 


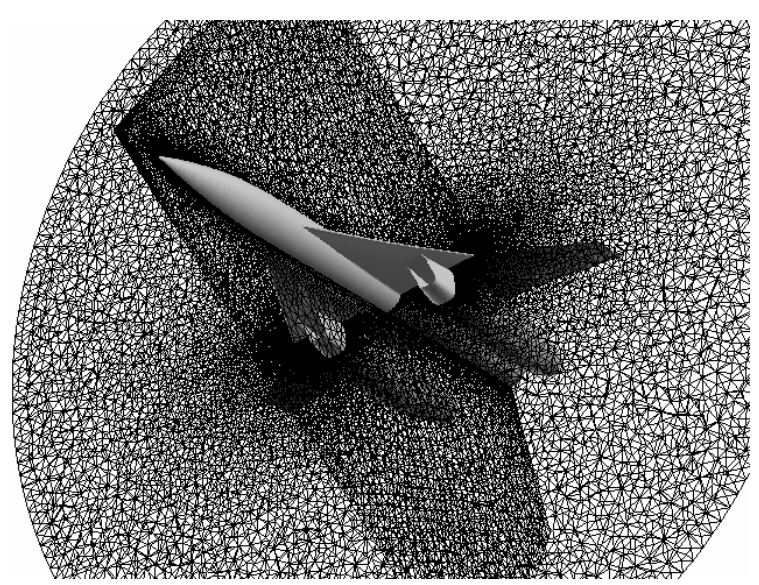

Fig. 6 Computational grid around a scaled supersonic airplane model

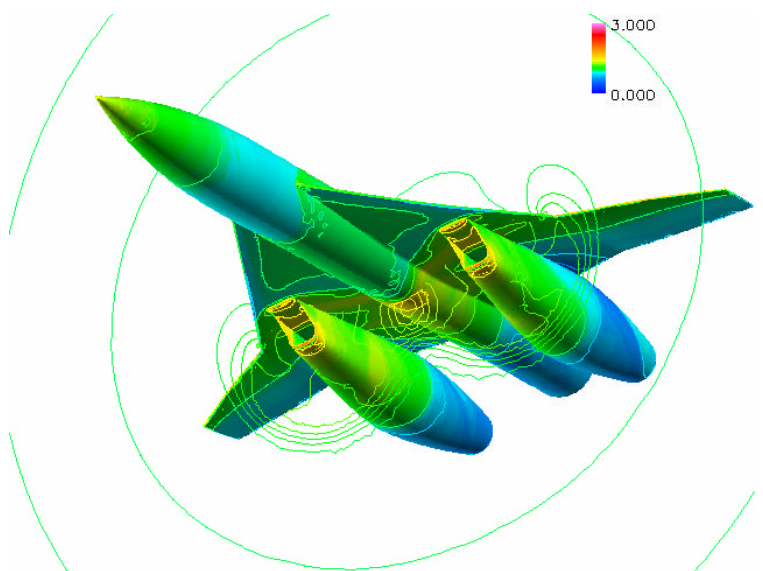

(a)

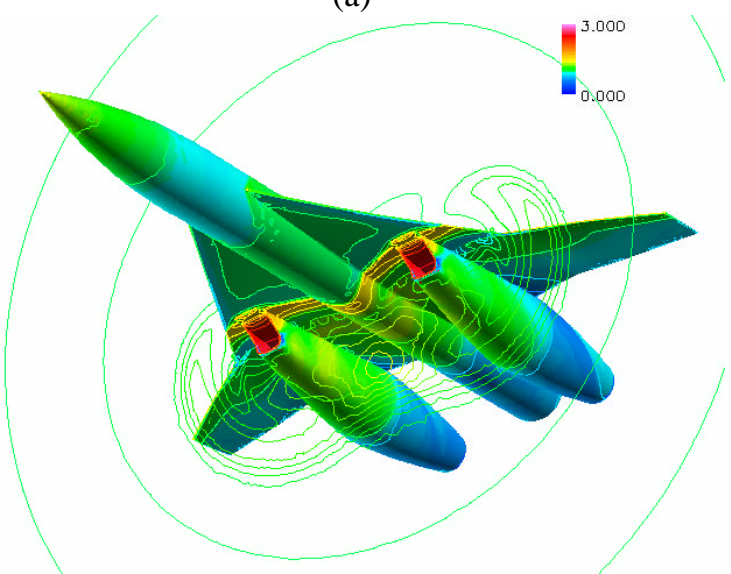

(b)

Fig. 7 Computed pressure contours around the wing-body-nacelle configuration (a) without bump (b) with bump

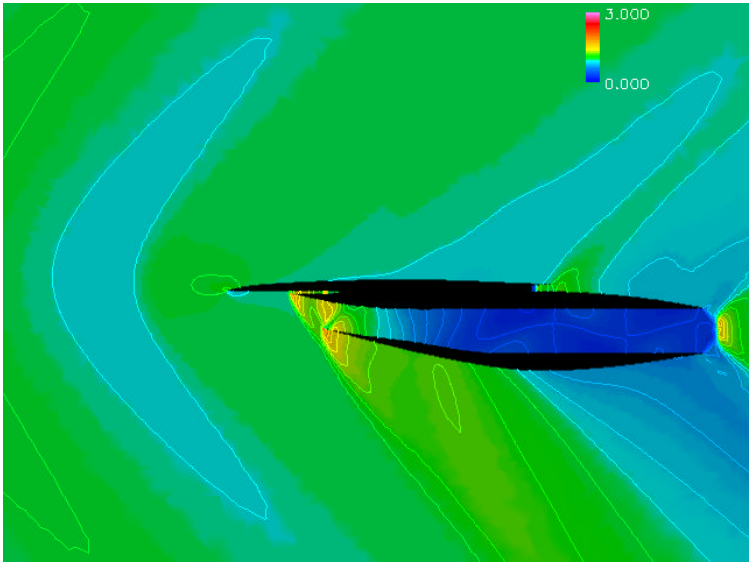

(a)

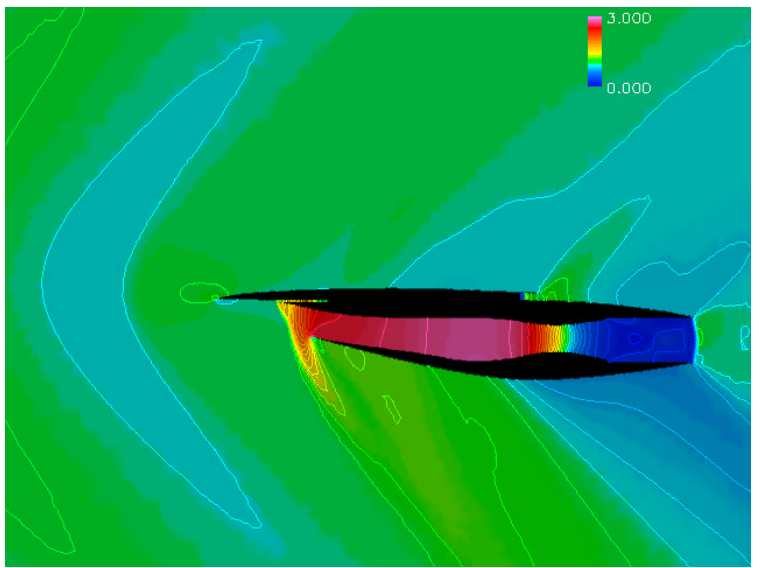

(b)

Fig. 8 Computed pressure contours cross sectional view of the nacelle (a) without bump (b) with bump

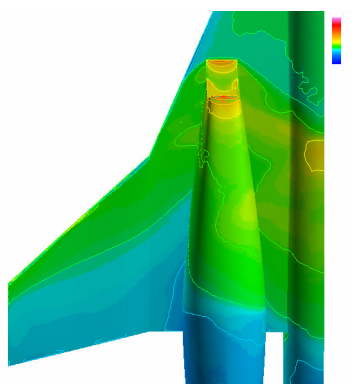

(a)

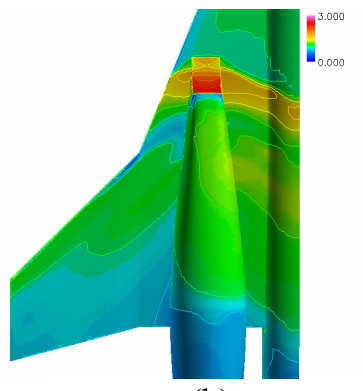

(b)
Fig. 9 Computed pressure contours bottom view (a) without bump (b) with bump

with experiment. The spillage drag does increase as the nacelle mass flow ratio decreases.

Furthermore, the viscous drag is estimated from its wetted area and turbulent boundary layer approximation. When the estimated viscous drag is added to the external pressure drag obtained from the present inviscid computations, the total drag shows excellent agreements with experimental data. 


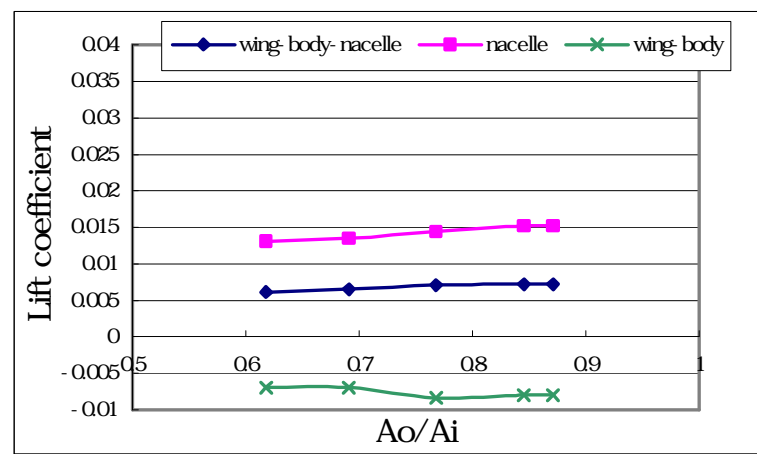

Fig. 10 Variation of lift coefficients vs. nacelle mass flow ratios

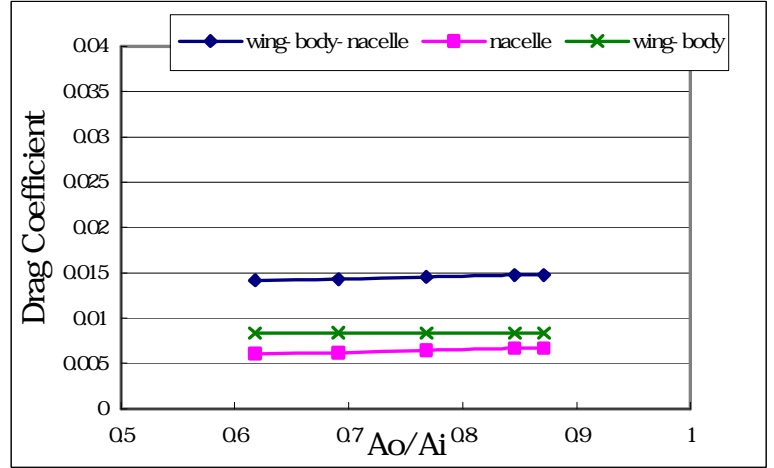

Fig. 11 Variation of drag coefficients vs. nacelle mass flow ratios

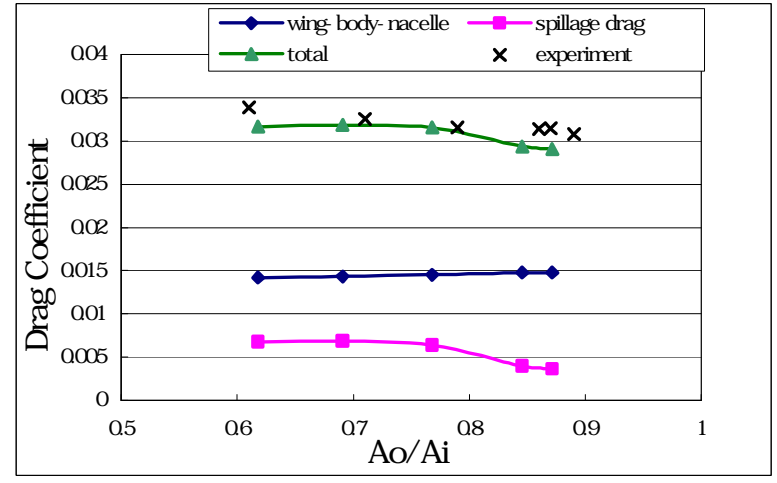

Fig. 12 Comparison of computational results with experiment including calculated spillage drag and estimated viscous drag

\section{Conclusion}

The numerical simulation of supersonic flows around the wing-body-nacelle configuration of the NAL scaled supersonic experimental airplane has been performed at various nacelle mass flow ratios by changing the bump heights inside the nacelle. In this study, flowfields were simulated by solving the
Euler equations with the unstructured grid method.

The external pressure drag was found to decrease as the nacelle mass flow ratio decreased, while the shock wave moved upstream and finally moved out from the inlet. The spillage drag was calculated and found to increase as the nacelle mass flow ratio decreased. Due to the spillage drag, the total drag is confirmed to increase as the nacelle mass flow ratio decreases. The computed drag agrees well with wind tunnel data obtained at NAL when the viscous drag estimation is added.

\section{Acknowledgements}

We would like to thank NAL supersonic experimental aircraft project team for providing the original geometry and its wind tunnel measurements. The calculations were performed using the supercomputer, ORIGIN2000 in the Institute of Fluid Science, Tohoku University.

\section{References}

[1] Shimbo, Y., Yoshida, K., Iwamiya, T. and Matsushima, K., "Aerodynamic Design of Scaled Supersonic Experimental Airplane," $1{ }^{\text {st }}$ SST-CFD Workshop, 1998, pp. 62-67.

[2] Shimbo, Y., Makino, Y. and Noguchi, M., "Wind Tunnel Test of the Powered National Supersonic Experimental Transport," the 48th Annual Meeting of the Japan Society of Fluid Mechanics, 2001, pp.84-93. In Japanese.

[3] Obayashi, S. and Guruswamy, G. P., "Convergence Acceleration of an Aeroelastic Navier-Stokes Solver," AIAA J., Vol. 33, No. 6, pp. 1134-1141, 1995.

[4] Venkatakrishnan, V., "On the Accuracy of Limiters and Convergence to Steady State Solutions," AIAA Paper 93-0880, 1993.

[5] Sharov, D. and Nakahashi, K., "Reordering of 3-D Hybrid Unstructured Grids for Lower-Upper Symmetric Gauss-Seidel Computations," AIAA J., Vol. 36, No. 3, 1998, pp.484-486.

[6] Jameson, A. and Turkel, E., "Implicit Schemes and LU Decompositions," Mathematics of Computation, Vol. 37, No. 156, 1981, pp.385-397.

[7] Yoshida, Y., Noguchi, M., Shimbo, Y., and Kuroda, F., "Comparison of Wind Tunnel Test and CFD Analysis on an Airframe/Nacelle Configuration of the Scaled Supersonic Experimental Airplane," the $39^{\text {th }}$ Aircraft Symposium, 2001, [CD-ROM]. In Japanese.

[8] Li, J., Li, F., and Qin E., "Numerical Simulation of Transonic Flow over Wing-Mounted Twin-Engine Transport Aircraft," Jounal of aircraft, Vol. 37, No. 3, 2000, pp. 469-478. 